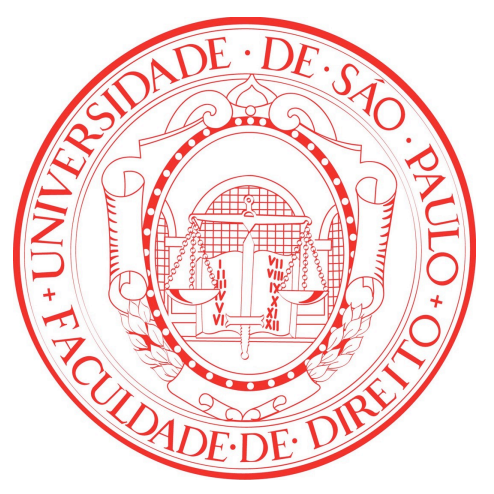

\title{
A INFLUÊNCIA DO DIREITO NAS DECISÕES DE FINANCIAMENTO DAS COMPANHIAS: UM ESTUDO DE CASO
}

\author{
DISSERTAÇÃO DE MESTRADO
}

Orientador: Professor Dr. Francisco SATIRo DE SOUZA Junior

UNIVERSIDADE DE SÃo PAULO

FACUldade De DiREITo do LARGo SÃo Francisco

SÃo PAUlo, SP 



\section{A INFLUÊNCIA DO DIREITO NAS DECISÕES DE FINANCIAMENTO DAS COMPANHIAS: UM ESTUDO DE CASO}

Dissertação apresentada à Banca Examinadora do Programa de Pós-Graduação em Direito, da Faculdade de Direito da Universidade de São Paulo, como exigência parcial para obtenção do título de Mestre em Direito, na área de concentração de Direito Comercial, sob a orientação do Professor Dr. Francisco Satiro de Souza Junior.

UNIVERSIDADE DE SÃo PAULO

FACULDADE DE DiREITO DO LARGo SÃo Francisco

SÃo PAULO, SP 
NAHAS, Fernando. A influência do direito nas decisões de financiamento das companhias: um estudo de caso. 2017. 114pp. Dissertação de mestrado - Faculdade de Direito da Universidade de São Paulo (FDUSP). São Paulo, 2017.

Orientador: Prof. Dr. Francisco Satiro de Souza Junior

Área de Concentração: Direito Comercial

Resumo: este trabalho demonstra uma das formas como o direito influencia escolhas de financiamento de empresas. Analisam-se as principais teorias sobre estrutura de capital e, em seguida, apresentam-se estudos empíricos que indicam o direito como um dos principais elementos externos às empresas que influenciam suas decisões de financiamento. Por fim, examina-se o caso real de uma companhia brasileira, confirmando os estudos teóricos e empíricos apresentados.

Palavras-chave: alternativas de financiamento; estrutura de capital; influência do direito; Oi S.A. 


\section{A INFLUÊNCIA DO DIREITO NAS DECISÕES DE FINANCIAMENTO DAS COMPANHIAS: UM ESTUDO DE CASO}

Dissertação apresentada à Banca Examinadora do Programa de Pós-Graduação em Direito, da Faculdade de Direito da Universidade de São Paulo, como exigência parcial para obtenção do título de Mestre em Direito, na área de concentração de Direito Comercial, sob a orientação do Professor Dr. Francisco Satiro de Souza Junior.

São Paulo, SP, de de 2017

\section{BANCA EXAMINADORA}



À minha querida avó Albertina.

Que saudades sinto da senhora! 


\section{AgRAdeCIMENTOS}

À Faculdade de Direito do Largo São Francisco e ao Colégio Bandeirantes, aos quais devo todas as minhas oportunidades e conquistas acadêmicas e profissionais.

À Professora Associada Dra. Mônica Herman Salem Caggiano e ao Professor Dr. Francisco Satiro de Souza Junior, por acreditarem em meu trabalho e por me concederem a oportunidade de aprofundar meu conhecimento e de buscar obter o título de Mestre em nossa querida Faculdade.

Ao Professor Titular Dr. Fernando Dias Menezes de Almeida, por aproximar nossa Faculdade à Universidade Jean Moulin Lyon 3. Graças a essa parceria, tive a honra de estudar direito francês na própria Lyon 3 e de coordenar junto a meus colegas um livro sobre o tema.

Ao Professor Dr. Cássio Cavalli e ao Professor Dr. José Alexandre Tavares Guerreiro, pelos apontamentos feitos em meu exame de qualificação.

Ao Professor Titular Dr. Dante Mendes Aldrighi, por sua disposição em aceitar um jurista em suas aulas de pós-graduação na FEAUSP e pelo auxílio em minhas pesquisas.

Aos meus pais, William e Silvia, pelo apoio incondicional.

Às minhas irmãs, Alessandra e Giovana, por caminharem ao meu lado.

A todos os meus primos, por me trazerem ao equilíbrio em meio a meus desafios acadêmicos e profissionais. 
"Sozinhos, vamos mais rápido. Juntos, vamos mais longe." 


\section{RESUMO}

NAHAS, Fernando. A influência do direito nas decisões de financiamento das companhias: um estudo de caso. 2017. 114pp. Dissertação de mestrado - Faculdade de Direito da Universidade de São Paulo (FDUSP). São Paulo, 2017.

Este trabalho demonstra uma das formas como o direito influencia as escolhas de financiamento que compõem a estrutura de capital de empresas. Num primeiro momento, analisam-se as principais teorias sobre estrutura de capital, a saber: Modigliani \& Miller, Pecking Order Theory, Trade-Off Model e teoria dos custos de agência. Em seguida, apresentam-se estudos empíricos que indicam o direito como um dos principais elementos externos às empresas que influenciam suas decisões de financiamento, concluindo que o direito, especificamente no que tange à proteção ao investidor e ao credor, é capaz de reduzir assimetria informacional e custos de falência e de agência (i.e. fatores identificados nas teorias estudadas), criando condições favoráveis para que os mercados financeiro e de capitais prosperem. Por fim, examina-se o caso real de uma companhia brasileira que balizou suas decisões de financiamento em função de questões ligadas a governança corporativa, confirmando, assim, a relevância do direito na determinação de sua estrutura de capital.

Palavras-chave: alternativas de financiamento; estrutura de capital; influência do direito; Oi S.A. 


\begin{abstract}
NAHAS, Fernando. The influence of law in the financing decisions of companies: a case study. 2017. 114pp. Master of Laws - Faculty of Law - University of São Paulo (FDUSP). São Paulo, 2017.
\end{abstract}

This dissertation demonstrates one of the ways through which the law influences the financing decisions that make up the capital structure of companies. First, the main theories on capital structure are analyzed, namely: Modigliani \& Miller, Pecking Order Theory, Trade-Off Model and agency cost theory. Next, empirical studies are presented, indicating law as one of the main elements external to companies that influence their financing decisions: specifically with regards to investor and creditor protection, law is capable of reducing informational asymmetry as well as bankruptcy and agency costs (as identified in the theories analyzed), by creating favorable conditions for the financial and capital markets to thrive. Finally, this work examines the real case of a Brazilian company that made its financing decisions based on corporate governance matters, thus confirming the relevance of the law in determining the capital structure of companies.

Keywords: financing decisions; capital structure; influence of law; Oi S.A. 
INTRODUÇ̃̃̃

1. TEORIAS ACERCA DA COMPOSIÇÃO DA ESTRUTURA DE CAPITAL.............14

1.1. Modigliani \& Miller........................................................................................

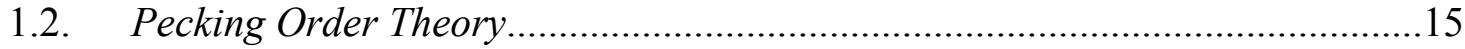

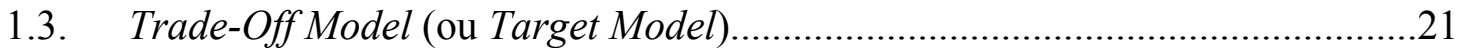

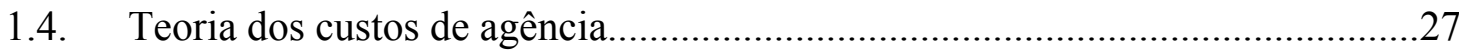

2. A INFLUÊNCIA DO DIREITO NO DESENVOLVIMENTO DOS MERCADOS FINANCEIRO E DE CAPITAIS E NAS DECISÕES DE FINANCIAMENTO............37

2.1. Direito e desenvolvimento dos mercados financeiro e de capitais.....................37

2.2. Rafael La Porta, Florencio López-de-Silanes, Andrei Shleifer

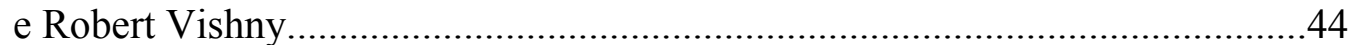

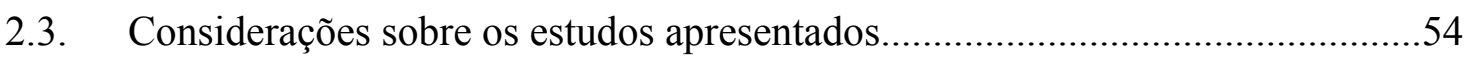

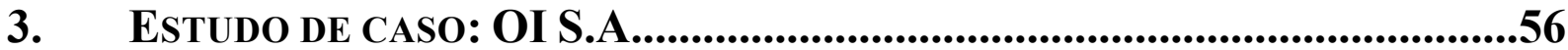

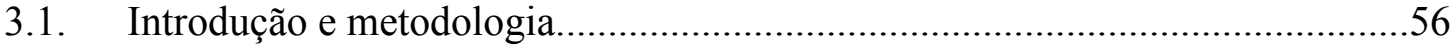

3.2. Entrada da Oi S.A. no mercado de telecomunicações brasileiro e fatos que marcaram seu histórico recente...................................................58

3.3. Contratação e emissão de dívidas.......................................................................68

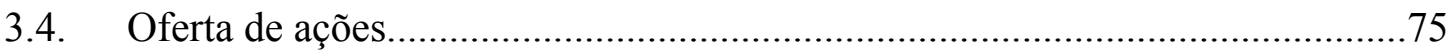

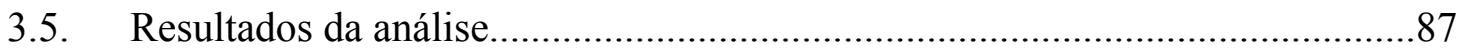

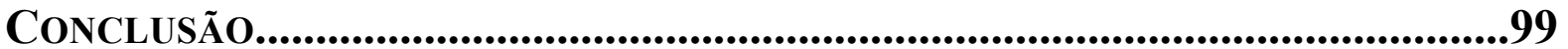

REFERÊNCIAS BIBLIOGRÁFICAS........................................................102 


\section{INTRODUÇão}

$\mathrm{O}$ direito e a economia analisam fenômenos semelhantes, muitas vezes coincidentes, mas sob óticas distintas. O jurista estuda como organizar e coordenar a elaboração e a interpretação de normas que regem determinada situação, traçando orientações sobre como um fenômeno específico é recepcionado pelo ordenamento jurídico em vigor. O economista, por sua vez, focaliza seus esforços em custos de transação, eficiência e incompletude contratual, dentre outros elementos vistos como incentivos a comportamentos oportunistas, à formação de cadeias produtivas e a outras condutas dos agentes econômicos.

Assim já antecipou SZTAJN (2014) ao indicar que juristas e economistas têm muito a explorar juntos, alavancando conhecimentos e desenhando uma arquitetura mais completa de institutos que, normalmente, são estudados de modo quase isolado por cada um desses dois grupos de acadêmicos. A normatização de um fenômeno econômico não pode se dar de forma plena sem a compreensão das consequências econômicas e dos custos sociais envolvidos, sem o exame dos porquês e dos para quês e sem o questionamento de como tornar mais eficiente a dinâmica entre os agentes envolvidos. Ao mesmo tempo, incentivos não podem ser devidamente analisados sem a compreensão das normas aplicáveis, dos princípios, da jurisprudência e do enquadramento jurídico que o ordenamento confere a um fenômeno em tela. Direito e economia caminham juntos, independentemente da visão de mundo ou orientação política adotada.

É altamente proveitoso que juristas e economistas dialoguem e trabalhem de modo conjunto para compreender e para criticar de forma construtiva as premissas, métodos, conclusões e a utilidade das normas, teorias e modelos. Isso resultará em aprofundamento e em melhor compreensão de fenômenos sociais, jurídicos e de mercado - se é que essas distinções não foram concebidas apenas para fins didáticos. No que tange às instituições ditas de cunho econômico-financeiro, mais especificamente, elas exercem papel chave na construção de um Estado próspero, estável e distributivo. Instituições financeiras, como bancos e bolsas de valores, e a poupança popular, todos sustentados por um sistema jurídico sólido e completo, são capazes de atender às demandas de mercado, canalizando os recursos dos investidores e provendo liquidez às companhias que demandam capital para projetos e empreendimentos específicos. 
Tais financiamentos podem ocorrer de diferentes formas e geram consequências distintas às companhias que arrecadam os recursos, particularmente no que concerne à estrutura de capital, isto é, à proporção entre capital próprio (i.e. dos sócios) e capital de terceiros utilizado no desempenho de suas atividades. Trata-se da ponderação entre, de um lado, financiamento interno, realizado com recursos oriundos da atividade da própria empresa ou resultantes de aportes dos sócios existentes, e, de outro, financiamento externo, que ocorre com a contratação de dívidas ou com a entrada de novos integrantes no quadro societário, como no caso de oferta pública de ações em bolsa de valores. Visando atender ao interesse social da companhia, os administradores devem buscar a estrutura de capital mais eficiente possível, de modo a diminuir custos totais de financiamento.

Por outro lado, a composição da estrutura de capital de determinada companhia é altamente conectada às relações entre seus "stakeholders", tanto no que tange a direitos patrimoniais, como no que tange a direitos políticos. Ações não apenas conferem o direito ao recebimento de dividendos, mas também, e principalmente, permitem ao acionista exercer influência sobre a administração por meio de seu poder de voto. No caso dos minoritários, por exemplo, seus direitos correspondem a uma forma de coagir administradores e controladores a tomar decisões alinhadas aos interesses da companhia e de todos os acionistas. Dívidas, por sua vez, não apenas correspondem a uma fonte de remuneração aos credores, que recebem juros: elas também podem impor aos tomadores uma série de condições a serem cumpridas ao longo da relação contratual, sob pena de excussão de garantias. A conclusão é a de que, diferentemente do que colocaram MODIGLIANI \& MILLER (1958), alterações na estrutura de capital de uma companhia implicam mudanças no jogo de poder entre, de um lado, os chamados "agentes internos" ou "insiders" (i.e. acionistas controladores e membros da administração) e, de outro, os “agentes externos" ou "outsiders" (i.e. credores e acionistas minoritários).

Esta temática assume ainda mais relevância se levada em conta a existência de conflitos de interesse entre agentes internos e externos, que podem resultar em expropriação por parte dos primeiros em relação aos últimos, seja pelo não pagamento de dividendos, pela dilapidação ou ocultação de patrimônio ou pela venda de ativos a preço abaixo de mercado a outras companhias sob o mesmo controle, seja também por meio do apontamento de membros da família sem a devida qualificação a cargos de administração, de remunerações exorbitantes a executivos ou do o uso da companhia para vantagens pessoais ("private benefits"). A depender da composição da estrutura de capital das empresas, o desalinhamento entre esses agentes assume contornos distintos, ora afetando 
principalmente a relação entre acionistas e credores, ora impactando mais significativamente a dinâmica entre controladores e não-controladores. Nesse sentido, como colocou LA PORTA et al. (2000, pp. 4), a governança corporativa corresponde em grande medida a um conjunto de direitos e obrigações (ou, em suas palavras, apenas "mecanismos") que protege investidores, como credores e minoritários, contra expropriações por parte dos "insiders" .

Esta é a problemática em que está inserido o presente estudo. Este trabalho se propõe a examinar o papel do direito nas escolhas de financiamento que compõem a estrutura de capital das empresas, focalizando sua atenção nos custos, conflitos e assimetrias que afligem a relação societária das companhias. Para tanto, realizam-se análises acerca do ponto de vista dos economistas em relação à função do direito na interação dos agentes econômicos e, em seguida, a partir de um caso concreto que evidencia conflitos entre controladores e não controladores na realidade brasileira, verificase uma das formas como esta influência do direito na estrutura de capital das empresas ocorre na prática.

Especificamente, esta dissertação está estruturada da seguinte forma: (i) no Capítulo 1, estudam-se as principais teorias acerca da estrutura de capital ótima, a saber, Modigliani \& Miller, Pecking Order Theory, Trade-Off Model e teoria dos custos de agência; (ii) no Capítulo 2, apresentam-se estudos empíricos que indicam o direito como um dos principais elementos externos às empresas que influenciam suas decisões de financiamento; combinando os Capítulos 1 e $2^{2}$, constrói-se a hipótese de que o direito, especificamente no que tange à proteção ao investidor e ao credor, corresponde a uma ferramenta de redução de assimetria informacional e de custos de falência e de agência entre agentes internos e agentes externos, criando condições favoráveis para que os mercados financeiro e de capitais prosperem; e (iii) no Capítulo 3, examina-se o caso real de uma companhia brasileira que balizou suas decisões de financiamento em função de

\footnotetext{
${ }^{1}$ Tradução livre de: "Corporate governance is, to a large extent, a set of mechanisms through which outside investors protect themselves against expropriation by the insiders".

${ }^{2}$ Os temas que escolhidos são aqueles que, segundo o julgamento do autor, são os mais frequentemente pesquisados em finanças corporativas e os que podem ajudar um estudante de graduação e de pós-graduação no estudo do assunto, tanto no contexto brasileiro, quanto no internacional. No entanto, não se pretende realizar uma revisão exaustiva da literatura, mas, sim, uma revisão seletiva e, portanto, sujeita a eventual viés do autor. É natural que o leitor considere que determinado artigo poderia ter sido incluído e esta limitação é reconhecida. O objetivo é o de demonstrar o quanto o tema é complexo, o de demonstrar que há muitos economistas pensando nessas questões e, acima de tudo, o de demonstrar que há diversas visões sobre os assuntos debatidos.
} 
questões ligadas a governança corporativa, particularmente decorridas de desgastes entre controladores e não-controladores, confirmando, assim, a relevância do direito na determinação de sua estrutura de capital. O trabalho é encerrado com conclusões sobre o tema e com sugestões de assuntos relacionados para pesquisas futuras. 


\section{CONCLUSÃo}

O Brasil vive hoje um capítulo complexo de sua história. Além da crise políticoeconômica, a crise institucional é patente e, de certa forma, a mais crítica. Para adquirir maturidade e estabilidade nos campos político, econômico, social e cultural, o país precisa focar na construção e na reforma de suas instituições. Para tanto, o diálogo entre o direito e a economia é fundamental e deve ser incentivado em todos os níveis. Fenômenos de mercado devem ser analisados de modo conjunto entre juristas e economistas, para que, caminhando lado a lado, sejam desenvolvidas soluções que se encaixem no ordenamento jurídico brasileiro, que sigam suas tradições, seus princípios, sua jurisprudência e que, ao mesmo tempo, funcionem como bons incentivos aos agentes, com baixos custos de transação e reduzidos conflitos de interesse.

Esta foi a linha que o presente trabalho seguiu ao longo de toda sua extensão e esta é sua contribuição. A análise foi iniciada com as principais teorias econômicas sobre estrutura de capital, que baseiam suas conclusões nos conceitos de assimetria informacional, custos de falência e conflitos de agência. Em seguida, realizou-se uma revisão de literatura focada no direito como um determinante no desenvolvimento dos mercados financeiro e de capitais. A partir dessas pesquisas, e de acordo com a linha teórica identificada, constatou-se que o direito possui o condão de reduzir a assimetria, os custos e os conflitos referidos, principalmente por meio da proteção ao investidor e ao credor, de modo a viabilizar o bom desenvolvimento dos mercados. Esta constatação foi, então, reforçada por meio do exame de um caso real envolvendo uma companhia brasileira que balizou suas decisões de financiamento em função de questões ligadas ao direito, particularmente à proteção a investidores e a credores.

Nessa situação prática, nos termos da Pecking Order, do Trade-Off Model e da teoria dos custos de agência (Capítulo 1), a Oi S.A. chegou ao limite de seu poder de financiamento, tendo em vista que, como alertaram La Porta et al., e outros autores (Capítulo 2), credores e acionistas minoritários não se sentiram suficientemente tutelados pelo direito para investir seus recursos nesta empresa. Nesse sentido, a falta de credibilidade de instituições jurídicas e o sentimento de vulnerabilidade no campo do direito resultaram na limitação dos agentes dispostos a alocar recursos na Oi S.A. e, então, na limitação das fontes de financiamento à sua disposição. Portanto, o caso real estudado exemplifica aspectos trazidos nos Capítulos 1 e 2 e aponta no sentido de que se, num 
primeiro momento, poderia se considerar que os administradores da companhia escolheriam sua fonte de financiamento livremente e apenas com base em critérios econômicos, financeiros e contábeis, na prática, suas decisões foram significativamente impactadas pela ordem jurídica vigente.

Assim, o presente estudo indica a relevância do direito para o desenvolvimento dos mercados financeiro e de capitais. É preciso não apenas prever direitos, mas principalmente garantir seu exercício, sempre com base em instituições sólidas, céleres, técnicas, especializadas e competentes. Sob o prisma dos trabalhos aqui analisados, esse corresponde ao caminho fundamental, coerente e consistente para expandir o mercado financeiro e de capitais, aumentando a oferta e o acesso ao financiamento externo, diminuindo os conflitos resultantes da concentração acionária, tornando mais eficiente a alocação de recursos, evitando que investidores sejam impactados por manobras de controladores e, finalmente, fazendo com que credores tenham seus direitos preservados em caso de inadimplemento dos devedores. Contrariando a concepção de que a ausência de regulamentação é a melhor forma de estimular a dinâmica de mercado, este estudo justifica políticas no sentido de proteger o investidor, indicando que elas podem ser favoráveis ao desenvolvimento dos mercados financeiro e de capitais como um todo.

O direito é, portanto, relevante na definição de estratégias de financiamento, cuja análise vai muito além de estudos puramente numéricos e objetivos. Percebe-se que aspectos exógenos a modelos financeiros exercem influência na tomada de decisões e que a percepção dos investidores em relação aos seus direitos e à proteção contra expropriações complementam análises das companhias em si, seus múltiplos, índices, custos, dentre outros. Tratam-se de aspectos que dificilmente podem ser quantificados, mas que impactam o desenvolvimento dos mercados financeiro e de capitais e que correspondem a elementos balizadores das decisões tomadas pelos investidores.

Caso os aspectos jurídicos destacados neste trabalho não sejam aprimorados, de nada servirão esforços como os que se têm conduzido no Brasil ao longo da última década. Se instituições jurídicas estão enfraquecidas, de nada serve, por exemplo, aumentar a quantidade de informações a serem obrigatoriamente divulgadas pelos participantes do mercado à título de cumprir com o princípio de "full disclosure". É preciso atribuir credibilidade às informações em si, mas também, e principalmente, ao próprio emissor e às instituições que zelam pelo mercado e que são juridicamente responsáveis por sua regulação. Aumentar a eficiência do ordenamento jurídico pode, inclusive, reduzir o 
envolvimento do Poder Judiciário na resolução das disputas, não só pela diminuição do número de litígios em si, mas também pelo aumento da importância de órgãos regulatórios e administrativos, como a CVM. Fortalecê-los fará com que especialistas e técnicos em mercado financeiro e de capitais analisem questões com mais experiência e proximidade, o que tende a aumentar a qualidade e a celeridade de suas decisões ${ }^{105}$.

Nesse sentido, a crescente internacionalização dos mercados pode ser de grande utilidade. Com dinamismo na atividade empresarial, crescente regulação e agilidade na troca de informações e de conhecimento entre mercados e agentes econômicos, permite-se não só a alocação de recursos em economias que se apresentem mais atrativas, mas também proporciona-se aos próprios reguladores e dirigentes o acesso a iniciativas e a projetos de melhoria conduzidos por outros países. Cria-se um ambiente de aprendizado mútuo, difundindo noções e princípios que podem ser favoráveis ao crescimento econômico, e incentivando ainda mais a adoção de reformas. Certamente, este é um campo a ser explorado em estudos futuros e que merece a atenção dos acadêmicos, tanto de juristas, quanto de economistas.

\footnotetext{
${ }^{105}$ A literatura costuma mencionar os casos da Polônia e da República Checa nos anos de 1990 para evidenciar a importância de normas e órgãos regulatórios bem estruturados no desenvolvimento da economia. À época, estes países apresentavam políticas econômicas, níveis de renda e qualidade do Poder Judiciário semelhantes, mas a forma como eles conduziram o desenvolvimento de seus mercados financeiro e de capitais foi distinta: enquanto a Polônia introduziu normas que visavam a divulgação de informações e a proteção a investidores e credores, com orgãos administrativos monitorando os participantes de mercado e com poderes para de fato regular o mercado, resultando em prosperidade no mercado acionário; a Republica Checa não conduziu reformas nesse sentido e, talvez em função disso, observou altos níveis de expropriação por parte de controladores e administradores e saída de empresas das bolsas de valores, resultando num mercado acionário quase inexistente (La Porta et al., 2000, pp. 22 e 23). Vide COFFEE, J. "The future as history: the prospects for global convergence in corporate governance and its implications". In: Northwestern Law Review, v. 93, pp. 631-707, 1999; PISTOR, K. "Law as a determinant of equity market development". In: MURRELL, Peter (ed.). The value of law in transition economies. Ann Arbor: University of Michigan Press, pp. 249-287, 1999; e GLAESER, E., JOHNSON, S., SHLEIFER, A. "Coase versus the Coasians". In: Quarterly Journal of Economics, v. 116 (3), pp. 853-899, 2001.
} 


\section{REFERÊNCIAS BIBLIOGRÁFICAS}

AGARWAL, Sumit \& MOHTADI, Hamid (2004). "Financial Markets and the Financing Choice of Firms: Evidence from Developing Countries". In: Global Finance Journal. n. 15, pp. 57-70, 2004. Disponível em http://faculty.apec.umn.edu/mohta001/debt-equity-103.pdf. Acesso em 8 de maio de 2014.

AGUILERA, Ruth V. \& WILLIAMS, Cynthia A. (2009). “'Law and Finance': Inaccurate, Incomplete, and Important". Forthcoming In: University Illinois Law \& Economics Research Paper No. LE10-002, 2009,14 pp. Disponível em http://poseidon01.ssrn.com/ delivery.php?ID $=48108512411110012510708811402412006410403509002900101011000$ 2117092118101096090113113041000008060051058110118087097065097082112060083 $\underline{0050010741020931160840000860250730590660030021120080260040260210841171250}$ $95102067076081068105009088126074113117116007 \& \mathrm{EXT}=$ pdf. Acesso em 6 de maio de 2016.

ARIDA, Persio \& BACHA, Edmar Lisboa \& LARA RESENDE, Andre (2004), "Credit, Interest, and Jurisdictional Uncertainty: Conjectures on the Case of Brazil". Forthcoming. Giavazzi F. \& GOLDFAJN (Ed.). Inflation Targeting and Debt: the Case of Brazil. Cambridge, MA: MIT Press, 2004, 25 pp. Disponível em: http://citeseerx.ist.psu.edu/viewdoc/ download?doi=10.1.1.532.9066\&rep=rep1\&type=pdf. Acesso em 2 de maio de 2016.

BARBOSA, Nelson (2017). "Taxa real de juro: evolução e perspectivas". Valor Econômico, 24 de fevereiro de 2017. Disponível em http://www.valor.com.br/cultura/4879800/taxa-real-de-juro-evolucao-e-perspectivas.

Acesso em 9 de junho de 2017.

BARROS, Lucas Ayres \& SILVEIRA, Alexandre Di Miceli da \& FAMÁ, Rubens (2005). "Does capital structure matter? Revisiting Modigliani and Miller's empirical work using Latin American and North American data". In: Latin American Business Review, v. 5, n. 3, pp. 43-64, 2005.

BAXTER, Nevins (1967). "Leverage, Risk of Ruin and the Cost of Capital". In: Journal of Finance, v. 22, n. 3, pp. 395-403, 1967.

BEDICKS, Heloisa Belotti (2009). Governança Corporativa e Dispersão de Capital: Múltiplos Casos no Brasil. São Paulo: Saint Paul, 2009. 202 pp. ISBN 9788598838847 (broch.).

BOOTH, Laurence \& AIVAZIAN, Varouj \& DEMIRGÜÇ-KUNT, Asli \& MAKSIMOVIC, Vojislav (2001). "Capital Structures in Developing Countries". In: Journal of Finance, v. 56, n. 1, pp. 87-130, 2001.

BRADLEY, Michael \& JARELL, Gregg A. \& KIM Han (1984). "On the existence of an Optimal Capital Structure: The Theory and Evidence”. In: Journal of Finance. v.39, pp. 857-880, 1984. 
BRANDÃO, Isac de Freitas \& CRISÓSTOMO, Vicente Lima (2015). “Concentração de propriedade e qualidade da governança da empresa brasileira". In: Revista Brasileira de Finanças (Online). Rio de Janeiro. v. 13, n. 3, julho, pp. 438-469, 2015. Disponível em https://www.google.com.br/url?sa $=t \& r c t=j \& q=\& e s r c=s \&$ source=web\&cd=1\&cad=rja\&ua $\mathrm{ct}=8 \& \mathrm{ved}=0$ ahUKEwjh oyfwKXUAhVDD5AKHTdEBr8QFgguMAA\&url $=\mathrm{http} \% 3 \mathrm{~A} \% 2$ F\%2Fbibliotecadigital.fgv.br\%2Fojs\%2Findex.php\%2Frbfin $\% 2$ Farticle $\% 2$ Fdownload $\% 2 \mathrm{~F}$ 45739\%2F56150\&usg=AFQjCNErJPHvOwiKc-2DldGifjdOW h2kg. Acesso em 4 de junho de 2017.

BRITO, Giovani Antonio Silva \& CORRAR, Luiz J. \& BATISTELLA, Flávio Donizete (2007). "Fatores Determinantes da Estrutura de Capital das Maiores Empresas que Atuam no Brasil". In: Revista Contabilidade \& Finanças. v. 18, n. 43, pp. 9-19, abril, 2007. Disponível em http://www.scielo.br/scielo.php?pid=S1519-70772007000100002\&script= sci arttext. Acesso em 15 de maio de 2015.

BRAU, James (2010). "Why do firms go public?" Working paper. Brigham Young University. Forthcoming. In: Oxford Handbook of Entrepreneurial Finance. Oxford University Press. 44 pp.

BRAU, James C. \& FAWCETT, Stanley E. (2006). "Initial Public Offerings: An Analysis of Theory and Practice”. In: The Journal of Finance. v. LXI, nº 1, Fev., 2006.

CAIXE, Daniel Ferreira \& KRAUTER, Elizabeth (2013). "A Influência da Estrutura de Propriedade e Controle sobre o Valor de Mercado Corporativo no Brasil". In: Revista Contabilidade e Finanças. São Paulo: USP. v. 24, n. 62, mai./jun./jul./ago., pp. 142-153, 2003. Disponível em http://www.scielo.br/pdf/rcf/v24n62/05.pdf. Acesso em 4 de junho de 2017.

CAMPOS, Taiane Las Casas (2006). "Estrutura da propriedade e desempenho econômico: uma avaliação empírica para as empresas de capital aberto no Brasil". In: Revista de Administração da Universidade de São Paulo. v. 41, n. 4, out./nov./dez., pp. 369-380, 2006.

CASAGRANDE NETO, Humberto \& SOUZA, Lucy A. \& ROSSI, Maria Cecília (2010). Abertura do Capital de Empresas no Brasil: um Enfoque Prático. 4. ed. rev. atual. São Paulo: Atlas, 2010. 138 pp. ISBN 9788522460625 (broch.).

CHISHOLM, Andrew (2002). An Introduction to Capital Markets: Products, Strategies, Participants. Chichester: John Wiley and Sons, 2002. 448 pp. (Wiley Finance Series). ISBN 0471498661 (enc.).

CINTRA, Marcos Antonio M. \& SILVA FILHO, Edison Benedito da (Org.) (2013). Financiamento das Corporações: Perspectivas do desenvolvimento Brasileiro. Livro 3. Brasília: IPEA, 2013. 231 pp. Disponível em http://repositorio.ipea.gov.br/bitstream/ 11058/2414/1/Livro \%20Financiamento\%20das\%20Corpora\%C3\%A7\%C3\%B5es\%20per spectivas $\% 20$ do $\% 20$ desenvolvimento $\% 20$ brasileiro.pdf. Acesso em 8 de maio de 2014.

CORREIA, Laise Ferraz \& AMARAL, Hudson Fernandes (2011). "Um índice de avaliação da qualidade da governança corporativa no Brasil". In: Revista Contabilidade e Finanças. São Paulo: USP. v. 22, n. 55, jan./fev./mar./abr., pp. 45-63, 2011. 
COTEI, Carmen \& FARHAT, Joseph \& ABUGRI, Benjamin A. (2011) "Testing trade-off and pecking order models of capital structure: does legal system matter?". In: Managerial Finance. v. 37.8, pp. 715-735, 2011.

COUTINHO, Luciano (2017). "O porquê dos juros altos". Valor Econômico, 10 de fevereiro de 2017. Disponível em http://www.valor.com.br/opiniao/4864602/o-porque-dosjuros-altos. Acesso em 9 de junho de 2017.

DE JONG, Abe \& KABIR, Rezaul \& NGUYEN, Thuy Thu (2007). "Capital Structure Around the World: The Roles of Firm- and Country-Specific Determinants". Erasmus University Working Paper, Rotterdam, Países Baixos, 2007.

DEL GIGLIO, Alex (2008). A Evolução do Mercado de Capitais e a Estrutura de Capital das Companhias Brasileiras. 69 f. Dissertação (Mestrado) - Escola de Administração de Empresas do Rio de Janeiro, Fundação Getúlio Vagas, 2008.

DELOITTE TOUCHE TOHMATSU (2013). Rumo à Abertura de Capital: Percepções das Empresas Emergentes sobre os Entraves e Benefícios. São Paulo: Deloitte Touche Tohmatsu, 2013.

DEMIRGÜÇ-KUNT, Asli \& LEVINE, Ross (2005). "Law and Firms' Access to Finance". In: American Law and Economics Review. v. 7(1), Spring, pp. 211-52, 2005.

DEMIRGÜÇ-KUNT, Asli \& MAKSIMOVIC, Vojislav (1998). "Law, Finance and Firm Growth”. In: Journal of Finance. v. 53, n. 6, pp. 2107-2137, 1998.

DEMIRGÜÇ-KUNT, Asli \& MAKSIMOVIC, Vojislav (1999). "Institutions, Financial Markets, and Firms Debt Maturity". In: Journal of Financial Economics. v. 54, pp. 295 336, 1999.

DEMIRGÜÇ-KUNT, Asli \& MAKSIMOVIC, Vojislav (2000). "Funding Growth in BankBased and Market-Based Financial Systems: Evidence from Firm-Level Data". World Bank Working Paper, 2432, 2000.

DONALDSON, Gordon (1961). "Corporate Debt Capacity". Division of Research Graduate. School of Business Administration. Harvard University, Boston, 1961.

DUTRA, Marcos Galileu Lorena \& SAITO, Richard (2002). "Conselhos de Administração: Análise de sua Composição em um Conjunto de Companhias Abertas Brasileiras". In: RAC: Revista de Administração Contemporânea. Curitiba. v. 6, n. 2, mai./ago., pp. 09-27, 2002. Disponível em http://www.scielo.br/pdf/rac/v6n2/v6n2a03.pdf. Acesso em 7 de junho de 2017.

EID JUNIOR, William (1996). "Custo e Estrutura de Capital: O Comportamento das Empresas Brasileiras”. In: RAE - Revista de Administração de Empresas. São Paulo, v. 36, n. 4, pp. 51-63, 1996. 
FAN, Joseph P. H. \& TITMAN, Sheridan \& TWITE, Garry (2012). “An International Comparison of Capital Structure and Debt Maturity Choices". In: Journal of Financial and Quantitative Analysis. v. 47, pp 23-56, 2012.

FERRAN, Eilís \& CHAN HO, Look. Company Law and Corporate Finance. Londres: Oxford University Press, 2014.

FRAGA NETO, Armínio (2017). "'O debate sobre os juros no Brasil', por Arminio Fraga Neto". O Globo, 10 de fevereiro de 2017. Disponível em https://oglobo.globo.com/economia/o-debate-sobre-os-juros-no-brasil-por-arminio-fraganeto-20903057. Acesso em 9 de junho de 2017.

FRANK, Murray Z. \& GOYAL, Vidhan K (2007). "Trade-off and pecking order theories of debt". In: ECKBO, Espen B. (Ed.). Handbook of Corporate Finance: Empirical Corporate Finance. Amsterdam: Elsevier. v. 2. Cap. 12. pp. 135-202, 2007.

GILSON, Ronald J. \& BLACK, Bernard S (1998). "Venture Capital and the Structure of Capital Markets: Banks Versus Stock Markets". In: Journal of Financial Economics. v. 47, n. 3, pp. 243-277, 1998.

GLEN, Jack \& PINTO, Brian (1994). "Debt or Equity? How Firms in Developing Countries Choose". Discussion Paper 22. Washington, DC: International Finance Corporation, 1994.

GORGA, Érica. "Changing the Paradigm of Stock Ownership from Concentrated Towards Dispersed Ownership? Evidence from Brazil and Consequences for Emerging Countries". Cornell Law School Working Paper. Setembro, 2008. Disponível em https://poseidon01.ssrn.com/delivery.php?ID $=554072120067119100096072095080031098$ $\underline{1020190850790490160850270111271220010270961180181221220530121040560550900}$ $\underline{2606810012002609200908605308301702708507609411408711906201705209311707506}$ $\underline{8103006001090013026114125016010003026093116026092098113111074012078 \& \mathrm{EXT}}$ $=$ pdf. Acesso em 17 de junho de 2017.

GRAHAM, John R. \& HARVEY, Campbell R. (2001). "The theory and practice of corporate finance: evidence from the field". In: Journal of Financial Economics. v. 60, pp. 187-243, 2001. Disponível em http://ac.els-cdn.com/S0304405X01000447/1-s2.0$\underline{\mathrm{S} 0304405 \mathrm{X} 0100044 \quad 7 \text {-main.pdf? tid }=\mathrm{a} 8 \mathrm{~d} 078 \mathrm{c} 8-\mathrm{db} 6 \mathrm{e}-11 \mathrm{e} 5-8 \mathrm{bf0}-}$ 00000aab0f01\&acdnat=1456370583 4a51d760e 8eb6db62c936742bfabf01b. Acesso em 26 de fevereiro de 2016.

HALE, Galina (2007). "Bonds or Loans? On the Choice of International Debt Instrument by Emerging Market Borrower". In: RES: Economic Journal - Royal Economic Society. v. 117, jan., 516, pp. 196-215, 2007. Disponível em http://onlinelibrary.wiley.com/doi/10.1111/ j.1468-0297.2007.02007.x/abstract. Acesso em 8 de maio de 2014. Acesso restrito.

HOLMSTRÖM, Bengt \& TIROLE, Jean (1993). "Market Liquidity and Performance Monitoring". In: The Journal of Political Economy. v. 101, n. 4. (Aug.), pp. 678-709, 2003. 
JAGGI, Bikki \& LOW, Pek Yee (2000). "Impact of Culture, Market Forces, and Legal System on Financial Disclosures". In: The International Journal of Accounting. v. 35, issue 4, pp. 495-519, 2000.

JENSEN, Michael C. \& MECKLING, William (1976). "Theory of the firm: managerial behaviour, agency costs, and capital structure". In: Journal of Financial Economics, v. 3, pp. 305-360, 1976.

JENSEN, Michael C. (1986). "Agency Cost of Free Cash Flow, Corporate Finance, and Takeovers". In: American Economic Review, v. 76, n. 2, May, pp. 323-329, 1986.

KORAJCZYK, Robert A. \& LEVY, Amnon (2002). "Capital Structure Choice: Macroeconomic Conditions and Financial Constraints". In: Journal of Financial Economics, v. 68, n. 1, pp. 75-109, 2002.

LA PORTA, Rafael \& LOPEZ-DE-SILANES, Florencio (1998). "Capital markets and legal institutions". In: BURKI, Shadid \& PERRY, Guillermo (coord.). Beyond the Washington consensus: institutions matter. Washington, D.C.: World Bank, 1998.

LA PORTA, Rafael \& LOPEZ-DE-SILANES, Florencio \& SHLEIFER, Andrei (2008). "The Economic Consequences of Legal Origins". In: Journal of Economic Literature, v. 46(2), pp. 285-332, 2008.

LA PORTA, Rafael \& LOPEZ-DE-SILANES, Florencio \& SHLEIFER, Andrei (2013). "Law and Finance After a Decade of Research". In: CONSTANTINIDES, George M. \& HARRIS, Milton \& STULZ, Rene M. (Ed.), Handbook of the Economics of Finance. V. 2, Part A, Chapter 6, pp. 425-491, 2013.

LA PORTA, Rafael \& LOPEZ-DE-SILANES, Florencio \& SHLEIFER, Andrei \& VISHNY, Robert (1997). "Legal Determinants of External Finance". In: The Journal of Finance. v. 52, (3), Julho, pp. 1131-1150, 1997.

LA PORTA, Rafael \& LOPEZ-DE-SILANES, Florencio \& SHLEIFER, Andrei \& VISHNY, Robert (1998). "Law and Finance". In: Journal of Political Economy. v. 106 (6), pp. 1113-1155, 1998.

LA PORTA, Rafael \& LOPEZ-DE-SILANES, Florencio \& SHLEIFER, Andrei \& VISHNY, Robert (2000a). "Investor protection and corporate governance". In: Journal of Financial Economics. v. 58, pp. 3-27, 2000.

LA PORTA, Rafael \& LOPEZ-DE-SILANES, Florencio \& SHLEIFER, Andrei \& VISHNY, Robert (2000b). "Agency Problems and Dividend Policies around the World". In: Journal of Finance. V. 55 (1), pp. 1-33, 2000.

LARA RESENDE, André (2017). “Teoria, prática e bom senso". Valor Econômico, 27 de janeiro de 2017. Disponível em: http://www.valor.com.br/cultura/4849060/teoria-pratica-ebom-senso. Acesso em 10 de março de 2017. 
LEAL, Ricardo Pereira Câmara (2008). "Estrutura de Capitais Comparada: Brasil e Mercados Emergentes". In: RAE: Revista de Administração de Empresas. São Paulo: FGV. v. 48, n. 4, pp. 67-78, out./dez., 2008.

LEAL, Ricardo Pereira. Câmara \& GOMES, Gabriel (2000). "Determinantes da estrutura de capital das empresas brasileiras com ações negociadas em bolsas de valores". In: LEAL, R. P. C.; COSTA JR. N. C. A.; LEMGRUBER, E. F. (Org.). Finanças corporativas. São Paulo: Atlas, 2000.

LEAL, Ricardo Pereira Câmara \& SAITO, Richard (2003). "Finanças Corporativas no Brasil". In: RAE: Revista de Administração de Empresas. São Paulo: FGV (eletrônica). v. 2, n. 2, jun./dez., 2003. Disponível em http://www.scielo.br/pdf/raeel/v2n2/v2n2a05. Acesso em 1 de junho de 2017.

LEAL, Ricardo Pereira Câmara \& CARVALHAL DA SILVA, André Luiz \& VALADARES, Silvia Mourthé (2002). "Estrutura de Controle das Companhias Brasileiras de Capital Aberto". In: RAC: Revista de Administração Contemporânea. Curitiba. v. 6, n. 1 , jan./abr., pp. 07-18, 2002. Disponível em http://www.scielo.br/scielo.php?script=sci arttext\&pid=S1415-65552002000100002.

Acesso em 1 de junho de 2017.

LISBOA, Marcos \& PESSOA, Samuel (2017). "Nada de novo no debate monetário no Brasil". Valor Econômico, 20 de janeiro de 2017. Disponível em http://www.valor.com.br/cultura/4842254/nada-de-novo-no-debate-monetario-no-brasil.

Acesso em 9 de junho de 2017.

LOWRY, Michelle \& SCHWERT, William (2002). "IPO Market Cycles: Bubbles or Sequential Learning?”. In: Journal of Finance. v. 67, n. 3, pp. 1171-1198, 2002.

LUCEY, Brian M. \& ZHANG, QiYu (2011). "Financial Integration and Emerging Markets Capital Structure". In: Journal of Banking \& Finance. v. 35, n. 5, 5.2011, pp. 1228-1238, 2011.

MAJLUF, Nicholas \& MYERS, Stewart (1984). "Corporate financing and investment decisions when firms have information that investors do not have". In: Journal of Financial Economics. v. 13, pp. 187-221. Disponível em http://www.nber.org/papers/w1396.pdf. Acesso em 30 de abril de 2016.

MILLER, Merton H. (1977). "Debt and taxes". In: Journal of Finance. v. 32, n. 2, May, 1977

MODIGLIANI, Franco \& MILLER, Merton (1958). "The Cost of Capital, Corporation Finance, and the Theory of Investment". In: American Economic Review. v. 48, n. 3, pp. 262-297, 1958. Disponível em: https://www2.bc.edu/ chemmanu/phdfincorp/MF891\%20 papers/MM1958.pdf. Acesso em 31 de maio de 2014.

MODIGLIANI, Franco \& MILLER, Merton (1963). "Corporate Income Taxes and the Cost of Capital: a Correction". In: American Economic Review. v. 53, n. 3, pp. 433-443, 1963. 
https://www2.bc.edu/ chemmanu/phdfincorp/MF891\%20papers/MM1963 .pdf. Acesso em 31 de maio de 2014.

MOODY'S (2009). Moody's assigns Aaa.br to Telemar's proposed debentures; upgrades Brasil Telecom to Baa2. Divulgado em 9 de dezembro de 2009. S/1; 4 pp. Disponível em: https://www.moodys.com/research/Moodys-assigns-Aaabr-to-Telemars-proposeddebentures-upgrades-Brasil-Telecom--PR 191671. Acesso em 20 de março de 2017. Acesso Restrito.

MOODY'S (2010). Moody's Assigns Baa2 Rating to Telemar Norte Leste Senior Unsecured Euro Bonds. Divulgado em 9 de dezembro de 2010. S/1; 3 pp. Disponível em: https://www.moodys.com/research/Moodys-Assigns-Baa2-Rating-to-Telemar-Norte-LesteSenior-Unsecured--PR 210278. Acesso em 20 de março de 2017. Acesso Restrito.

MOODY'S (2011a). Moody's atribui rating Baa2 a BRL 1,0 bilhão em títulos com vencimento em 5 anos da Brasil Telecom. Divulgado em 8 de setembro de 2011. S/l; 3 pp. Disponível em: https://www.moodys.com/research/ Moodys-atribui-rating-Baa2-a-BRL10-bilho-em-ttulos--PR 225714. Acesso em 20 de março de 2017. Acesso Restrito.

MOODY'S (2011b). Moody's atribui ratings Baa2/Aaa.br para emissão de BRL 2,35 bilhões debêntures seniores sem garantia de ativos reais com vencimento em 7 anos da Brasil Telecom. Divulgado em 23 de dezembro de 2011. S/l; 3 pp. Disponível em: https://www.moodys.com/research/Moodys-atribui-ratings-Baa2Aaabr-para-emisso-deBRL-235-bilhes--PR 234376. Acesso em 20 de março de 2017. Acesso Restrito.

MOODY'S (2012a). Moody's atribui rating Baa2 para emissão de USD 1,0 bilhão em notas seniores sem garantia de ativos reais com vencimento em 10 anos da Brasil Telecom. Divulgado em 6 de fevereiro de 2012. S/l; 3 pp. Disponível em: https://www.moodys.com/research/Moodys-atribui-rating-Baa2-para-emisso-de-USD-10bilh o--PR 236856. Acesso em 20 de março de 2017. Acesso Restrito.

MOODY'S (2012b). Moody's atribui ratings Baa2/Aaa.br para emissão de BRL 2,0 bilhões em debentures seniores sem garantia de ativos reais da Brasil Telecom. Divulgado em 14 de fevereiro de 2012. S/l; 3 pp. Disponível em: https://www.moodys.com/research/Moodys-atribui-ratings-Baa2Aaabr-para-emisso-deBRL-20-bilhes--PR 237775. Acesso em 20 de março de 2017. Acesso Restrito.

MOODY'S (2012c). Moody's afirma todos os ratings Baa2/Aaa.br da Telemar Norte Leste e da Brasil Telecom. Divulgado em 27 de fevereiro de 2012. S/1; 3 pp. Disponível em: https://www.moodys.com/research/Moodys-afirma-todos-os-ratings-Baa2Aaabr-daTelemar-Norte-Leste--PR 238734. Acesso em 20 de março de 2017. Acesso Restrito.

MOODY'S (2012d). Moody's coloca ratings da Oi S.A. em revisão para possível rebaixamento. Divulgado em 16 de agosto de 2012. S/l; 3 pp. Disponível em: https://www.moodys.com/research/Moodys-coloca-ratings-da-Oi-SA-em-reviso-parapossvel--PR 252860. Acesso em 20 de março de 2017. Acesso Restrito.

MOODY'S (2012e). Moody's rebaixa os ratings da Oi para Baa3/Aal.br; perspectiva negativa. Divulgado em 31 de outubro de 2012. S/l; 3 pp. Disponível em: 
https://www.moodys.com/research/Moodys-rebaixa-os-ratings-da-Oi-para-Baa3Aa1brperspe ctiva-negativa--PR 258827. Acesso em 20 de março de 2017. Acesso Restrito.

MOODY'S (2013a). Moody's: Resultados do 1T da Oi estão em linha com as expectativas. Divulgado em 2 de maio de 2013. S/l; 1 pp. Disponível em: https://www.moodys.com/ research/Moodys-Resultados-do-1T-da-Oi-esto-em-linha-com--PR 272341. Acesso em 9 de agosto de 2014. Acesso Restrito.

MOODY'S (2013b). Credit Opinion: Oi S.A. Divulgado em 7 de outubro de 2013. S/l; 1 pp. Disponível em: https://www.moodys.com/research/Oi-SA-Credit-Opinion-COP 600053026. Acesso em 9 de agosto de 2014. Acesso Restrito.

MOODY'S (2013c). Rating Action: Moody's affirms Oi's Baa3/Aal.br rating with a negative outlook following announced merger with Portugal Telecom. Divulgado em 2 de outubro de 2013. S/1; 1 pp. Disponível em: https://www.moodys.com/research/Moodysaffirms-Ois-Baa3Aa1br-rating-with-a-negative-outlook-following--PR 283488. Acesso em 26 de março de 2017. Acesso Restrito.

MUNHOZ, Eduardo Secchi (2009). "Desafios do direito societário brasileiro na disciplina da companhia aberta: avaliação dos sistemas diluído e concentrado". In: CASTRO, Rodrigo R. Monteiro de \& ARAGÃO, Leandro Santos de (Coord.). Direito Societário Desafios Atuais. São Paulo: Quartier Latin, 2009, pp. 119-155.

MUNHOZ, Eduardo Secchi (2010). "Transferência de Controle nas Companhias sem Controlador Majoritário". In: CASTRO, Rodrigo R. Monteiro de \& MOURA AZEVEDO, Luís André N. de (Coord.). Poder de Controle e Outros Temas de Direito Societário e Mercado de Capitais. São Paulo: Quartier Latin, 2010, pp. 285-324.

MUNHOZ, Eduardo Secchi (2015). "Financiamento e Investimento na Recuperação Judicial". In: CEREZETTI, Sheila C. Neder \& MAFFIOLETTI, Emanuelle Urbano (coords.). Dez Anos da Lei no 11.101/2005. São Paulo: Almedina, 2015, pp. 264-290.

MYERS, Stewart (1984).“The Capital Structure Puzzle”. In: Journal of Finance. v. 39 (July 1984), pp. 575-92, 1984.

MYERS, Stewart C. (2001). "Capital Structure”. In: Journal of Economic Perspectives. v. 15, issue 2, pp. 81-102, 2001.

NAHAS, Fernando. "Estruturas de Capital e Alternativas de Financiamento: Um Paralelo entre Oferta de Ações e Emissão de Dívidas". 2014. 70pp. Trabalho de Conclusão de Curso (Master in Financial Economics / CEAFE) - Escola de Economia de São Paulo da Fundação Getulio Vargas (FGV/EESP). São Paulo, 2014. Orientador: Prof. Guido Marcelo Borma Chagas.

NAKANO, Yoshiaki (2017). “Aritmética monetarista desagradável”. Valor Econômico, 02 de fevereiro de 2017. Disponível em http://www.valor.com.br/opiniao/4855902/aritmeticamonetarista-desagradavel. Acesso em 9 de junho de 2017.

NOVAES, Ana (2000). "Privatização do setor de telecomunicações no Brasil". In: PINHEIRO, Armando Castelar \& FUKASAKU, Kiichiro (org). A Privatização no Brasil: 
o Caso dos Serviços de Utilidade Pública. Rio de Janeiro: BNDES-OCDE. Fev. 2000. 34 pp. Disponível em: http://www.bndes.gov.br/SiteBNDES/export/sites/default/bndes pt/ Galerias/Arquivos/conhecimento/ocde/ocde05.pdf. Acesso em 20 de março de 2017.

PAGANO, Marco \& PANETTA, Fabio \& ZINGALES, Luigi (1998). "Why Do Companies Go Public? An Empirical Analysis". In: Journal of Finance, v. 53, n. 1, pp. 27-64, 1998.

PAGANO, Marco \& ROËL, Ailsa (1998). "The Choice Of Stock Ownership Structure: Agency Costs, Monitoring and the Decision to Go Public". In: The Quartely Journal of Economics. $\quad$ v. 113. pp. 187-225, 1998. Disponível em https://www.researchgate.net/publication/24091603 The Choice Of Stock Ownership S tructure Agency Costs Monitoring And The Decision To Go Public. Acesso em 17 de junho de 2017.

PEROBELLI, Fernanda Finotti Cordeiro \& FAMÁ, Rubens (2002). "Determinantes da estrutura de capital: aplicação a empresas de capital aberto brasileiras". In: Revista de Administração. São Paulo. v. 37, n. 3, pp. 33-46, jul./set, 2002.

PEROBELLI, Fernanda Finotti Cordeiro \& FAMÁ, Rubens (2003). "Fatores determinantes da estrutura de capital para empresas latino-americanas". In: RAC: Revista de Administração Contemporânea. v. 7, n. 1, pp. 9-35, 2003.

PITTA, André Grünspun (2017). Aspectos jurídicos do processo de formação de capital por meio do mercado de valores mobiliários: As alternativas de organização e capitalização das sociedades empresárias. 493 pp. Doutorado - Faculdade de Direito da Universidade de São Paulo (FDUSP). São Paulo, 2017.

PROCIANOY, Jairo laser \& SCHNORRENBERGER, Adalberto (2004). "A influencia da estrutura de controle nas decisões de estrutura de capital das companhias Brasileiras". In: Revista Brasileira de Economia. Rio de Janeiro. v. 58, n.1, jan./mar., 2004.

RAJAN, Raghuram Govind \& SERVAES, Henri (2003). "The effect of market conditions on initial public offerings". In: Venture capital contracting and the valuation of hightechnology firms. Oxford University Press, 2003. Disponível em: http://citeseerx.ist.psu.edu/viewdoc/download;jsessionid=26D797C2F6B742CFB281F747

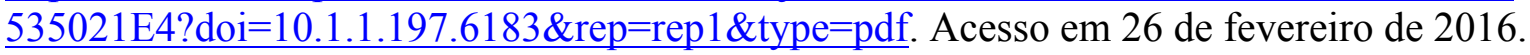

RITTER, Jay R. (1991). “The Long-Run Performance of Initial Public Offerings”. In: Journal of Finance. v. 46, Issue 1 (Mar.), pp. 3-27, 1991.

RITTER, Jay R. (2011) “Equilibrium in the IPO Market”. In: Annual Review of Financial Economics. v. 3, pp. 347-374, 2011.

RUDGE, Luiz Fernando \& CAVALCANTE, Francisco \& MISUMI, Jorge Yoshio (2009). Mercado de Capitais: o que é, como funciona. 7. ed. rev. atual. Rio de Janeiro: Elsevier, 2009. 395 pp. ISBN 9788535226188 (broch.).

SCHIEHLL, Eduardo \& SANTOS, Igor Oliveira dos (2004). "Ownership structure and composition of boards of directors: evidence on Brazilian publicly- traded companies". In: 
Revista de Administração. São Paulo. v. 39, n. 4, pp. 373-384, out./nov/dez. 2004. Disponível

em https:/www.google.com.br/url? sa $=t \& r c t=j \& q=\& e s r c=s \&$ source $=$ web $\& c d=2 \& c a d=r j a \& u a$ $\mathrm{ct}=8 \& v e d=0$ ahUKEwjBy 7 mtra3UAhWDnJAKHWEmBdcQFggwMAE\&url=http $\% 3 \mathrm{~A} \% 2$ F\%2F200.232.30.99\%2Fdownload.asp\%3Ffile\%3DV3904373ap384.pdf\&usg=AFQjCNG ElpjT1Bq-yZArWcK8g2CRhwYCIg. Acesso em 7 de junho de 2017.

SILVEIRA, Alexandre Di Miceli da \& BARROS, Lucas Ayres B. de C. \& FAMÁ, Rubens (2008). "Atributos corporativos e concentração acionária no Brasil". In: RAE: Revista de Administração de Empresas [online]. São Paulo. v. 48, n. 2, pp. 51-66, abr./jun., 2008. Disponível em http://www.scielo.br/pdf/rae/v48n2/v48n2a05.pdf. Acesso em 7 de junho de 2017.

SPAMANN, Holger. "Law and Finance' revisited” (2008). Harvard Law School John M. Olio Center Discussion Paper No. 12, 2008. Disponível em http://www.law.harvard.edu/ programs/olin center/fellows papers/pdf/Spamann 12.pdf. Acesso em 7 de maio de 2016.

STANDARD \& POOR'S (2014). Perspectiva dos ratings da Klabin alterada para negativa refletindo aumento na alavancagem; Ratings ' $B B B{ }^{-'}$ ' 'brAAA'. S/l; Acesso Restrito, 4 pp.

SZTAJN, Rachel (2014). "Ronald H. Coase e a importância de perguntar". In: Revista de direito empresarial: ReDE. São Paulo: Revista dos Tribunais, v. 2, n. 1, pp. 201-209, jan./fev., 2014.

TITMAN, Sheridan \& WESSELS, Roberto (1988). "The determinants of capital structure choice". In: Journal of Finance. v. 43, n. 1, mar., pp. 1-19, 1988.

ZINGALES, Luigi (1995). "Insider Ownership and the Decision to Go Public". In: The Review of Economic Studies. v. 62, n. 3, pp. 425-448, 1995. 